\title{
L'armoire de la mémoire : la parole enclose
}

\section{Danielle Bohler}

\section{OpenEdition}

Journals

Édition électronique

URL : https://journals.openedition.org/clo/1212

DOI : $10.4000 /$ clo. 1212

ISSN : 2266-1816

Éditeur

INALCO

\section{Édition imprimée}

Date de publication : 1 janvier 2007

Pagination : 19-36

ISBN : 978-2-85831-174-3

ISSN : 0396-891X

\section{Référence électronique}

Danielle Bohler, «L'armoire de la mémoire : la parole enclose », Cahiers de littérature orale [En ligne], 62 I 2007, mis en ligne le 16 mars 2013, consulté le 24 juillet 2021. URL : http://journals.openedition.org/ clo/1212; DOI : https://doi.org/10.4000/clo.1212

Ce document a été généré automatiquement le 24 juillet 2021.

\section{(c) (†) 8)}

Cahiers de littérature orale est mis à disposition selon les termes de la Licence Creative Commons Attribution - Pas d'Utilisation Commerciale 4.0 International. 


\title{
L'armoire de la mémoire : la parole enclose
}

\author{
Danielle Bohler
}

\section{NOTE DE L'AUTEUR}

Ces réflexions sont issues d'une série d'enquêtes menées sur les Prologues dans les récits médiévaux, le paratexte où le lecteur se trouve « inscrit » comme destinataire privilégié, avec l'effet de réel d'une « identité » d'auteur vu pour ainsi dire « de face et de profil », dans son geste d'écriture, après la découverte d'un livre.

$1 \mathrm{Au} \mathrm{XII}$ e siècle une scène intime inaugure le Conte de Floire et Blancheflor : "l'autre jour ", entré en une chambre, un vendredi après le repas, le narrateur prêtait l'oreille à ce que racontaient deux dames. Elles parlaient d'amour, l'aînée racontait à sa sœur une histoire vieille de deux siècles, qui lui avait été transmise par un clerc qui l'avait lue dans un livre. Raconté avec grâce - «avenanment » - le récit emboîte des voix tirées d'un livre et entendues. Il s'engage vers la rédaction de l'œuvre nouvelle. Un savoir qui va d'une écoute à une parole issue d'un dire, ce dernier issu d'un livre d'où l'a tiré un homme de savoir : la complexité de la transmission ancre le récit à venir, sans lourdeur, dans le suspens d'une narration dont les acteurs sont de très jeunes amants, séparés par l'infortune, mais promis à un bonheur sans fin. Ce seuil de l'œuvre invite à explorer le motif du livre, l'objet d'amour tellement présent dans la littérature médiévale qu'il en apparaît comme le fétiche : le livre tiré d'une armoire, concrète ou métaphorique, délie une parole pour les futurs lecteurs et auditeurs.

2 En ces temps-là, la lettre était rare, toujours ancrée à une main qui la trace, aux instants d'un projet, à l'éphémère d'un geste. $\mathrm{Du} \mathrm{XII}^{\mathrm{e}}$ au Xve siècle, cette aventure de la durée conquise est relatée par tant de récits qu'elle en devient obsédante, comme si le péril d'être ou de n'être pas guettait sans cesse ce produit raffiné des activités humaines : le livre. 
3 L'ère Gutenberg était encore lointaine : pendant quelques siècles la littérature en langue vernaculaire, tout comme les textes latins, restera tributaire d'une page parcourue de signes, de la composition d'un feuillet, de parchemin ou de papier. Même si les copies successives ont su en garantir l'existence, ou se multiplier sous l'effet d'un succès, le livre garde le statut d'un témoin unique. À chaque fois il affirme la tâche d'un copiste et d'un atelier : pour ces temps-là, il n'est de page qui ne soit liée à la main qui lui a donné vie.

4 Attachée à l'événement d'une inscription manuelle, dans les murs supposés d'un atelier, la lettre pouvait rester lettre morte, dès lors qu'elle se dérobait, enfouie bien souvent dans une "armoire ». Ce secret est suggéré avec tant d'insistance, jusqu'à la veille de la Renaissance, qu'il apparaît comme le fétiche énigmatique d'une culture disparue, l'emblème obsessionnel, sacralisé peut-être et parfois redouté. Objet d'un attachement singulier, par de multiples formes et circuits narratifs, le livre échappe à la trivialité qui sera son sort à partir de l'abondance imprimée. Il se prête donc à l'imaginaire de la transmission: un récit guetté dans une chambre, tiré d'un livre ancien dans Floire et Blancheflor, ou un "livret» trouvé en un lieu oublié, "en nonchalloir ", en quête d'existence et d'identité.

5 Une dynamique double, si l'on envisage le champ textuel médiéval : de la voix au livre, du livre à la voix nouvelle, l'écrit est assumé, objet d'amour de celui qui pourra, grâce à la trouvaille, prendre voix. Ainsi, dans cet objet devenu banal à nos yeux, décèle-t-on l'obsession d'une naissance, une passion pour les causalités mystérieuses, pour les phases du secret et les temps de latence, somme toute un trésor de mémoire assigné à une gestation autonome due au temps lui-même. Rien ne dit mieux que ce parchemin oublié le trajet de la mémoire aux mots pour la dire : la culture manuscrite comme parole qui s'ouvre à une révélation langagière.

6 La spatialisation du livre n'est pas indifférente, et le réceptacle aura belle fortune au fil du temps. «En un des livres de l'aumaire », l'histoire trouvée dans un des livres de la bibliothèque de l'église Saint-Pierre à Beauvais, écrivait Chrétien de Troyes dans le prologue de Cligès. Un lien étroit se noue entre le livre et l'armoire qui rime avec "mémoire "; si l'armoire est susceptible de délivrer le livre, le "livre " à son tour apparait comme l'« armoire » des secrets et leur conservatoire. Pour évoquer l'intimité discrète des sentiments, on pourra évoquer les "secrettes aumaires » des coeurs (Baumgartner, 1999).

\section{Le temps de la lecture}

7 Scènes de charme et fait culturel, séduisant le regard par une focalisation, esquissant une "sociabilité de la culture » ainsi qu'un ressort romanesque (Cerquiglini-Toulet, 2006, 13), l'abondance des gestes de lecture - $\mathrm{du} \mathrm{XII}^{\mathrm{e}}$ au Xv $\mathrm{xv}^{\mathrm{e}}$ siècle - illustre l'usage du livre et de sa valeur comme motif d'exception. Acte polymorphe, qu'il s'agisse d'une lecture solitaire, à deux ou pour un groupe, en un " cercle familial ou curial », la lecture crée son propre espace, celui de la rêverie. La jeune lectrice suscite «l'ennamourement» du narrateur. La lecture partagée, dans un espace symbolique préservé, favorise le geste de désir et la fusion amoureuse, pour les amants de Rimini, pour Héloïse et Abélard. 
8 Dans d'autres scènes de lecture, vivantes par l'acquisition d'un savoir et la quête des éléments dont il faut tirer parti au terme d'une étude de « livres ", le déroulement d'un nouvel écrit est donné avec précision. Dans le prologue de Mélusine, au moment où le libraire Coudrette prend la parole, le mystère du lignage se trouve déjà bien fixé par plusieurs manuscrits et une véritable tradition, une bibliothèque de mémoire. Le seigneur de Parthenay lui donne un modèle, concernant le passé légendaire de l'ancêtre Mélusine. Pour exécuter l'ordre de mettre en vers les sources consignées, le « libraire » rapporte qu'on a trouvé, dans la tour de Maubergeon, deux beaux livres en latin, à l'authenticité reconnue, qu'on a fait traduire. Quelques mois plus tard, la même histoire lui a été confirmée par le comte de Salisbury, possédant lui aussi un livre sur le château de Lusignan, exactement le même récit que les deux précédents. Enfin, à l'issue du roman lui-même, c'est encore la lecture d'une inscription déchiffrée qui découvre la grande boucle lignagère: Geoffroy la Grande Dent s'engouffre dans la caverne des ancêtres, découvrant une chambre souterraine, admirable de beauté. Un gisant, une statue, une riche tombe, et une dame très belle qui tient une tablette, aussi éloquente qu'un livre : «Geoffroy s'émerveille à cette lecture » et elle l'emplit de joie (Coudrette, 117-119). L'empreinte dans le roc, la caverne découverte constituent une belle métaphore du creuset de la mémoire, difficile d'accès et garante de la durée ${ }^{1}$.

Mais la fonction du livre est plus subtilement encore invoquée par l'abondance des scènes de trouvailles. La découverte du livre tout comme le livre ouvert pour un temps intime est un événement culturel et symbolique d'un grand prix. Sa genèse fait l'objet d'un rituel narratif tout particulier; au premier chef le mystère de la parole léguée, mission d'une particulière gravité, pour inscrire dans le temps de l'écrit l'événement à consigner. Le grand ensemble arthurien repose sur ces médiations mémorielles, dont la plus frappante est la mission de Merlin confiée à Blaise dans la forêt. L'obsession de la fixation empreinte de mystère ne peut se saisir que dans un contexte culturel où le livre est rare. Tout converge vers la fixation par l'écrit, passant par la voix qui doit nourrir le livre, l'instaurant comme haut lieu de la mémoire.

Le confesseur de la mère de Merlin, sur l'ordre de celui-ci, est informé de tout, à commencer par le témoignage de la sagesse remarquable de l'enfant. Ainsi Blaise commence-t-il à écrire sous la dictée un livre qui relatera les enseignements sacrés et l'histoire du Graal et de ses possesseurs. Il est envoyé en Northumberland, région forestière, où il vivra tout adonné à la tâche du prophète qui viendra le trouver et lui racontera ce qu'il faut pour la rédaction de son livre. Ce qui procurera au scribe la joie éternelle : cette histoire dictée et consignée sera célèbre parmi toutes, et le scribe connaîtra la grâce. Pour que Blaise puisse s'engager dans sa mission, Merlin lui ordonne de chercher suffisamment d'encre et de parchemin, car les faits sont nombreux, qui vont de l'histoire du Christ jusqu'à Joseph d'Arimathie, au Saint-Graal, à la stratégie des diables décidant d'engendrer un homme par la ruse. Les étonnantes révélations sont recueillies avec vigilance, et Blaise rassemblera le livre de Merlin et le livre de Joseph: "Les deux livres, une fois réunis, feront un très beau livre et l'un et l'autre seront d'égale valeur ", assure le prophète.

$11 \mathrm{Au}$ début de la Mort Artu, Arthur fait rassembler et consigner les nouvelles malheureuses de la fin de la Quête du Saint Graal. Mais auparavant déjà, la voix de la collecte se faisait vive dans le Lancelot en prose :

Einsinc com Lanceloz ot dites ses aventures furent eles mises en escrit, et pour ce que li fet estoient greingnor que nus de çaux de laienz, les fist li rois mestre par lui seul si que des fez et des ovres trova l'an .I. grant livre en l'aumaire le roi Artu après 
ce qu'il fu navrez a mort en la bataille Mordret, si com li contes vos devisera apertement.

(Lancelot, t. IV, LXXXIV 72) Perceval, afin de lui remettre un livre autographe que le petit prophète lui a jadis confié » (Koble, 2006, 128). Pour ce retour sur sa petite enfance Merlin fait donc se rencontrer le quêteur du Graal et un personnage issu de l'histoire ancienne du roman. C'est ainsi que l'ermite Helias remet à Perceval « le livre de la science» de Merlin. Le héros retardera la transmission du livre inconnu et engage le vieillard à raconter sa jeunesse. Enfin, il révèle son identité « en échange du livret » que Perceval remettra à Blaise après la mort du vieillard.

L'épisode repose donc sur un dispositif complexe, très travaillé, qui exploite un procédé classique d'amplification, associant le topos de l'attente et les vertus dilatrices du quiproquo pour produire du récit.

(Koble, 2006, 131)

L'amplification suggère ainsi « une réflexion sur les origines du cycle ». Le livre est un objet précieux, puisque « le livret de Merlin, protégé comme une relique dans un petit 'coffinet' a pris la place du Graal, 'vaissel' musical » (Koble, 2006, 137)2.

Si les récits arthuriens sont ainsi attachés à l'évocation de leur propre enfance, on rappellera que l'acte de lecture engage fréquemment l'enfant et son identité. Geoffroy lisait le déroulement d'une généalogie glorieuse et mystérieuse ; d'autres se verront, par la lecture, relater leur naissance, leur conception, l'histoire des parents. Tel Milun chez Marie de France, dont le destin est programmé dans la lettre qu'écrit sa mère au moment où elle doit confier à d'autres mains l'enfant dont l'origine se découvre à l'adolescence grâce à une lecture essentielle. Ainsi les mères, que l'on croit perdues, programment soigneusement la révélation de l'origine, et elles font connaître au père les éléments qui permettront de nouer les liens avec leur fils (Milun, vers 220-46). Lettre ou livre, la vérité est portée et médiatisée par le signe tracé. De la voix à l'écrit, du livre contenu au creux de la voix, destiné à faire naître la voix, l'écrit fétiche apparaît lié à la construction de la personne.

Sans oublier la fonction du livre qui vaut comme parole d'autorité, porteur d'un «bel sen ». Dans le roman de Robert de Blois, pour souligner l'importance de son message, la mère de Beaudous met en abyme la parole antérieure. En se référant à l'autorité du 
livre, elle programme l'incognito de son fils, au moment où il s'engage sur son itinéraire héroïque :

Biaus fiz, fait ele, encor te wel

Un bel sen dire ; onques en fuel

De parchemin ne fu escris

Plus bez, q'aussi bes ne soiz is.

(Beaudous, vers 1-4)

Elle lui dicte la rétention de son nom. Cette stratégie imaginée par la mère s'inquiète des valeurs d'une idéologie. Mieux vaut porter un nom masqué. Le nom «Chevalier aux deux écus » appartient au domaine important des marques distinctives, en vérité un «bel sen» dont elle répétera l'importance. Désigner, cacher, révéler appartiennent à une même logique symbolique. La mère insiste sur la sociabilité et l'importance de l'amitié, sur le contrôle de la parole et du chant importun, et surtout sur le fait que « Li nons ne fait pas la proesse » au vers 43 , d'où la nécessité d'arborer une marque distinctive qui occulte le nom :

Por ce dous escuz porteras

Ensemble joins, et si diras

Ce qui tu es demande nuns :

Li chevaliers as dous escus.

(Beaudous, vers 280-4)

Enfin, à l'objet manuscrit sont attribuées des fonctions métaphoriques à la fois passagères et fondamentales, tel le rêve d'un livre disponible et déployé au regard, porteur de connaissance. Ainsi, l'appel à la métaphore du parchemin parcouru situe l'itinéraire spatial du Chemin de longue étude chez Christine de Pizan. La voie où elles sont entrées, lui dit la Sibylle, se déroule aussi facilement qu'un parchemin, et cette voie est destinée aux lettrés qui veulent parcourir le monde sans rechercher une route trop ardue (Chemin de longue étude, v. 932 et suivants).

19 On voit donc à l'œuvre cette interaction et cette dialectique constante entre les activités orales et écrites dont parlait J. Goody (1994:13) : elles sont récurrentes dans le champ textuel médiéval, par la symbolique des parcours, de l'identité en gestation, arrachée au risque de l'oubli. Ce trajet passe par la voix, qui ne peut faire l'économie du signe tracé.

\section{Les grains de poussière}

«Lire, c'est aller à la rencontre d'une chose qui va exister »: Calvino était très justement cité au seuil d'une enquête sur l'histoire de la lecture (Manguel, 1998, 41). Si la lecture suscite un état favorable, par une sorte de rêverie, l'écriture y puise son origine : l'acte d'écrire le livre reçoit son droit à l'existence. Dans les scènes de lecture solitaire chez Christine de Pizan, l'entrée en écriture se fait subtilement. La topique s'éclaire de l'histoire de la culture et d'une observation de l'homme au livre. Tissage de motifs hérités, la topique permet ici encore des structures d'emboîtement, comme si la parole écrite ne pouvait dépendre que d'un trésor enfoui, attendant une voix nouvelle.

21 Nombreux sont les textes qui font usage du motif de l'énigme d'une parole enclose dans un espace interdit ou inconnu: le moment de la trouvaille est un temps fort. Elle permet, dans le Roman de Troie au XII ${ }^{\mathrm{e}}$ siècle, de signaler la dette des auteurs, qui nomment leurs modèles «et parfois même s'y réfèrent dans le corps de l'œuvre »: l'auteur se montre volontiers comme « chasseur de livres» (Baumgartner, 1999, 1). La 
fiction du manuscrit trouvé assure l'horizon d'une authenticité. Dans le roman antique, le livre est découvert accidentellement dans une "armoire " à Athènes; le manuscrit est transféré à Rome, et d'une langue à l'autre, du grec en latin. Ce qui permet à l'auteur de travailler désormais dans le domaine anglo-normand. Ainsi, le manuscrit trouvé

reste sans doute le fondement à partir duquel se construit le nouveau texte. Ce «prétexte » reste indispensable pour rappeler ce que sait fort bien le Moyen Âge, qu'aucun texte n'apparaît ex nihilo, et l'on pourrait tout aussi bien voir dans les mentions de ce livre source une manière de désigner l'hypotexte en perpétuelle expansion que constitue à partir du XIIIe siècle l'ensemble des romans arthuriens et avec lequel tout nouvel auteur est obligé de composer, à tous les sens du mot.

(Baumgartner, 1999, 11)

Autre témoin de l'ampleur du champ textuel où le motif est ancré, le Haut Livre du Graal où le texte latin à l'origine du récit en langue romane est trouvé sur l'île d'Avalon, dans la bibliothèque d'une "sainte abbaye sise à l'extrémité des Marais Aventureux" (Strubel, 2007, 1053) $)^{3}$. Au XIV ${ }^{\mathrm{e}}$ siècle, le Roman de Perceforest confirme l'imaginaire de l'origine. Le comte Guillaume de Hainaut s'est fait remettre en 1308 par l'abbé de l'abbaye de Wortimer un manuscrit "scellé dans une très vieille tour et composé en grec ». Ce manuscrit aurait été traduit du grec en latin par un clerc d'Angleterre, ce texte latin est ensuite traduit en français par un moine. Entre oralité et écriture en effet, le livre une fois découvert devient objet de récit, car le savoir nouveau en nourrit la dynamique. Indispensable à l'écriture, l'objet-fétiche apparait comme une relique à décoder, une riche et forte trace qu'il faut s'approprier. Ainsi, l'enchâssement de l'écrit à décrypter s'adressera-t-il au regard, à l'oreille, à la parole multipliée. Que le livre apparaisse comme noyau d'une transmission collective - de la consignation imposée par Merlin à celles qu'ordonne Arthur, pour une diffusion curiale, une "aurality" (Chinca et Young, 2005, 2) $)^{4}$ ou comme nourriture goûtée dans le secret, pour la genèse d'une plume d'auteur, ce qu'affirment tant de prologues du Moyen Âge tardif, l'objet est un lieu caché, dont l'ouverture engage à une filiation. Savouré dans le silence d'une bibliothèque, ou inscrit dans une parole nouvelle, le livre scelle le lancement d'une œuvre, dans une archéologie du geste et d'une délivrance.

23 À rebours, on devine la menace que l'écrit ne puisse se faire si, en amont, le manque à exister ne l'avait menacé. C'est le cas des scènes de manuscrits trouvés, d'écrits enfouis, explicitement victimes de l'oubli. Si le livre est un précieux conservatoire, il risque toujours l'indifférence des hommes. Nombreux sont les scénarios qui assignent au livre, au " grimoire » tiré de l'« armoire », le statut d'un objet du hasard, pourtant essentiel à la naissance d'une œuvre. Comme un trésor virtuellement porteur de tous les risques de l'oubli, et du bonheur de la découverte. Il s'agit d'une fouille sous la poussière, bien plus précise que dans le Roman de Troie au XII ${ }^{\mathrm{e}}$ siècle. Le seuil de l'œuvre est la trouvaille d'un livre, dont la parole réappropriée est lancée pour un nouveau devenir.

La raison de sa perte est ignorée. Égaré, négligé, «mis en nonchalloir», le livre recouvert de poussière était relégué, à l'écart de toute transmission, dans l'armoire du temps, sous les gravats des années. Parfois, enfin, un livre à la langue vieillie et désuète est le signe du temps qui passe, autre poussière qui occulte l'entendement des hommes. La voix enclose fait place à celle du maître d'œuvre d'un nouveau texte. Le $\mathrm{xv}^{\mathrm{e}}$ siècle insistera sur la découverte intime du lecteur, plongé dans le livre trouvé, fasciné par l'« aventure » qu'il y découvre. Comme le schème d'une mémoire ravivée désormais, et donc promise à la vie. 

inaugurale fait place à une apparition étrange, à une rencontre singulière qui à son tour participe d'un mythe de l'origine : la rencontre des dames allégoriques chez Christine de Pizan invite à la technicité du livre, l'encre et la truelle de sa plume, le ciment, le mortier et la belle pierre: "prends donc la truelle de ta plume et hâte-toi de bien maçonner et d'œuvrer avec ardeur », lui dit Dame Raison (chap. xIV). Ailleurs, l'héritage revendique la filiation reconnue. Dans le prologue du Roman de Jason de Raoul Lefèvre, auprès de Philippe le Bon, la figure étrange de Jason sortant de la brume exige que soit ressuscité un savoir. "Ancre ta galee, et prends ta plume ", enjoint le héros accablé de tristesse à l'auteur qui, en une pose identitaire, vient de se décrire, l'esprit ballotté tel un navire en quête d'un port paisible. Le personnage qui surgit oriente ainsi la trame de son livre, et la tradition est désignée de façon frontale : il faut écrire, comme dit Jason, « ce dont tu as leu la verité ». De la sorte, sans ambages, s'esquisse le geste donné à voir, la main tendue vers l'œuvre du passé, le souvenir avivé d'un livre lu, attendant d'être rendu à la vie, le mouvement d'un regard qui interroge, puis se dirige vers la plume.

Énonciation rhétorique, poétique du livre? En une pose convenue, l'auteur occupe un espace visant l'effet de réel : il s'est trouvé, un jour, un lecteur assez curieux pour fouiller la poussière, y porter un regard avide, mû par le désir croissant de découverte. Espace clos d'une bibliothèque, ou espace d'un repli sur soi, enrichi d'une temporalité limitée au prologue, autant d'éléments qui inscrivent le texte dans le moment incertain de sa naissance. Le Moyen Âge du xve siècle est attaché à cette représentation du livre, à l'anecdote personnelle d'un lecteur, anonyme ou connu, se penchant sur le livresource ; attaché à ce moment privé, voire onirique, proche du secret d'une genèse. La topique du moment singulier, du bras tendu et d'un regard de découvreur a pour effet un suspens lié au hasard et à la contingence. L'auteur préside au livre à venir : il esquisse des gestes qui valent comme signaux. La main et le regard, événements de peu d'importance et gestes convenus, n'occultent jamais qu'ils sont la parabole d'un rapport au passé et à l'oubli.

Ainsi le prologue est-il une sorte d'ouvroir, une fabrique du texte. Dans l'espace de la bibliothèque, l'écrivain instaure l'acte de naissance du livre : soit le manuscrit, oublié dans la poussière, cet enfant perdu dans l'anonymat d'une foule de livres, recevant le statut d'objet de mémoire digne de donner naissance au texte nouveau; soit la perception d'une figure culturelle, surgissant au cœur d'une rêverie qui engage à la mission de la plume, à la création d'un livre.

Au seuil de la fiction, au sein d'un espace privé, dans une sorte de tête-à-tête avec les folios couverts de signes, se décrivant en train de feuilleter des pages, le narrateur instaure le texte comme objet d'un futur labeur. De face, de profil, l'auteur devient le point focal, fouillant les strates du passé, en un geste lié au hasard. Si la focalisation sur la stature de l'écrivain était déjà frappante chez Christine de Pizan, dans la vacuité un peu morne d'une heure de lecture, elle est plus timide pour le rédacteur anonyme de Jean d'Avesnes, parlant de soi dans une scène où, "par adventure ", pour passer le temps, il goûte un moment de loisir «en l'estude d'un tresnoble seigneur, garny a planté de pluiseurs biaulx livres », des « livrez a souhait », parmi lesquels il fouille : «Je quis finablement tant que je m'arestay a ung ancien livre en latin qui contenoit pluiseurs hystoirez", un livre "comme mis en nonchalloir", et il parcourt ces «pluiseurs hystoirez », pour s'arrêter à l'une d'elles qui mérite particulièrement son attention (Jean d'Avennes, 41). 

librairie ${ }^{5}$, ou l'auteur anonyme de Gillion de Trazegnies passant en une abbaye, et tel autre encore saisi par l'activité de lecture, en train de parcourir " pluiseurs volumez et traittiez ", s'arrêtant " a ung livret qui fait mencion des haultez entreprisez, amours et beaulx fais d'armes d'un conte d'Artois, dont la narracion se fera cy apprez » (Roman du comte d'Artois, 151), Pierre Sala enfin, mettant ses lunettes pour déchiffrer des lettres usées. Au seuil de l'œuvre, la perception prend pour objet le recueil de folios saisi par celui qui en sera l'auteur et le narrateur, grâce à cette temporalité du seuil qui lui confère une identité topique. Le livre à venir requiert en effet une main humaine tournant les pages: le locuteur veut s'inscrire dans le temps, en train de prendre connaissance de sa source, par la mise en abyme d'une temporalité du livre lui-même, oublié des hommes. Au terme d'un choix, comme il est dit dans le prologue d'Isaÿe le Triste, où le narrateur dit s'être mis au travail « apres avoyr tourné et revolvé plusieurs livres et anciens romans $»^{6}$.

Cette trouvaille dans l'espace d'une bibliothèque peut être précisée, dans Gillion de Trazegnies particulièrement, par l'arrivée du narrateur à l'abbaye de Hainaut où il pose une question sur le secret des tombes, deux corps de femmes entourant le corps d'un homme. L'objet mortuaire va libérer un savoir qui deviendra le roman lui-même : la raison d'être des sépultures engendre celle du livre. Les morts sont rendus à la vie d'un destin, en même temps que le livre est redonné à la lecture. Le locuteur apparaît comme témoin oculaire, à la fois des lieux et des pages découvertes. Dans l'espace d'une bibliothèque, le topos engage le corps et sa gestuelle : l'auteur du prologue devient le narrateur de sa propre anecdote de lecteur. La chaîne des agents de mémoire apparaît comme scène d'une origine nouvelle.

L'appareil du prologue met progressivement l'accent sur le livre, objet de consommation sociale, paradoxalement limité à une scène de hasard. Au seuil de l'œuvre, cette anecdote peut donc être évaluée en termes d'identité auctoriale. À leur manière, les scénarios appartiennent à la gamme des mystères possibles : non plus le manuscrit scellé dans une vieille tour, mais un livre trouvé au milieu d'autres livres, parfois tendu par la main d'un ami, fruit d'un bon hasard, comme dit le narrateur dans le prologue de Guillaume de Palerne :

A ceste occasion, par aulcun mien amy, fut a moy, humble translateur et traducteur de la presente hystoire, presenté l'ancien livre auquel elle [l'histoire] estoit contenue quasi comme en frische, en grant danger d'estre perdue, anichilee et enrouillee d'oubly.

(Guillaume de Palerne, prologue)

Le processus d'actualisation d'une lecture engage souvent un jugement sur le support de la transmission, soit le livre abîmé et lacunaire, dont les lettres se dérobent, soit la langue vieillie et démodée, la langue usée par le temps. Certes, la langue "ornée " appartient à la topique: pourtant l'évaluation d'une langue peu compréhensible, desservant le propos et impropre à la communication désirée, ramène au jugement d'une subjectivité identitaire. Le «je» est en acte dans cette démarche : l'usage de l'imparfait retrace l'inscription du locuteur dans la scène, le processus d'une lecture difficile à laquelle se heurte le visiteur de la bibliothèque. La restriction au champ personnel du lecteur - évaluant à la fois l'intérêt de l'œuvre trouvée, la qualité qu'il en devine, les difficultés linguistiques - concerne le regard d'un individu. Victime des ans, le livre s'offre au rajeunissement de la langue, mais l'évaluation de sa qualité appartient au lecteur-découvreur d'un texte rare, digne d'être transmis à la mémoire (Régnier- 
Bohler, 1989, 187-213). En même temps s'impose le lecteur nouveau, le public à qui le livre est destiné, ces «lisans » encore souvent associés, en un groupe binaire, aux « escoutans », aux « délicates oreilles » (Guillaume de Palerne, prologue). Ainsi, pour celui qui parle du livre à naître, l'œuvre possède la dignité d'un matériau apte à l'édification, apte aussi à la séduction d'une esthétique, par une langue mieux adaptée à la lecture, embellie pour le plaisir.

Pour l'automne du Moyen Âge, qui voit prospérer les imprimeurs, le labeur de la plume est ici rapproché des gestes agricoles, garants d'une mémoire vigilante. Le livre originel est en sommeil, tout comme la terre asséchée. Les métaphores végétales sont mises au service du travail. Semblable à un organisme de la nature, l'œuvre requiert l'effort des hommes :

Tout ainsi comme la vigne qui n'est de toutes façons labouree facillement est subjecte a mauvaises herbes et si elle n'est bien et convenablement taillee le fruit en est moins savoureux, si la convient amender et ameliorer, aultrement facillement demourroit en frische, ainsi est des hystoires antiques et choses dignes de memoire, qui sont proffitables et savoureuses comme le bon vin.

(Guillaume de Palerne, prologue)

Redire le texte avec des mots neufs se compare aux soins attentifs, garants d'une terre fertile: le prologue d'Isaÿe le Triste affirme que les hauts faits livrés à l'oubli ressemblent à la « vieille racine de l'arbre qui de jour en jour pert son honneur radical par faulte d'estre arrousee et cultivee en sa vertu primeraine ». Écarté le risque de la terre inféconde, le livre accorde la promesse du plaisir.

Dans la mise en prose de La Belle Hélène de Constantinople, Jean Wauquelin dit avoir suivi «le contenu d'un livret rimé » que lui a donné son seigneur, "pour retrenchier et sincoper les prolongacions et motz inutiles qui souvent sont mis et boutez en telles rimes »(Wauquelin, 2002, 14). La rouille dont parle Pierre Durand - l'œuvre « enrouillee d'oubly " - est peut-être l'effet de la langue vieillie du "rommant antique rymoié en sorte non intelligible ne lisible». Au début du Roman de Richart sans paour, Gilles Corrozet parle d'un " petit livre ", "nouvellement translaté de vieille rime en prose", qu' 'il eust esté impossible de le translater nattement pour le langage corrompu dont il estoit plain». Nettoyer, rénover appartiennent aux intentions énoncées par un locuteur qui parle de la peine prise et de l'imperfection de sa tâche. À quoi peut s'ajouter la mention de son propre plaisir, ce que fait le narrateur du Roman de Floriant et Florete: «... pour ce que la matiere du present livret m'est plus agreable a lire en prose que en rime, me vueil pener de le transporter de rime en prose» $(1983,1)$.

Le trouveur de livre apparaît comme le favori du hasard. Réflexe culturel, la topique parle d'un rapport à l'objet écrit, à un moment où le geste de la main et l'homme à sa table inaugurent les récits, et où la bibliothèque entre amplement dans le monde de la représentation. Souvenir d'une mise en abyme dans la longue durée, la trouvaille du livre, ainsi que l'apparition de la figure mythologique dont le destin a été lu, sont connotées ici des gestes de l'étonnement, du parcours des yeux, pour lesquels un locuteur assume, dans une scène qu'il dit singulière, une fonction centrale. Le geste d'une découverte personnelle, circonscrite dans le temps, conforte le rapport de filiation avec l'amont: le livre à découvrir, le destin à déchiffrer pour que s'opère la genèse d'un texte neuf. la performance curiale et les pratiques de la lecture, désormais au cœur de la recherche 
actuelle : la lecture engage une poétique et meut l'imaginaire (Bouchet, 2008). Objet culturel privilégié par l'invention nouvelle, le livre intègre l'ensemble des signes symboliques qui parsèment le champ textuel médiéval. Lire signifie décrypter un secret, dont l'un des plus subtils apparait par la trouvaille, chez Marie de France, de la baguette de coudrier autour de laquelle s'enlace le chèvrefeuille. L'inscription résume, condense le temps des rencontres et du bonheur : l'écorce écrite sert de récit vivant. La connaissance différée par l'acte de lecture assure la transmission, au même titre que l'émission vocale. Mais le silence de l'objet, la lecture différée d'une écorce ou d'un livre sous la poussière nourrissent le suspens de la découverte. Il n'est d'histoire que secrète et cryptée. N'y aurait-il pas ici un mythe de l'origine du livre, reposant sur l'impérieuse nécessité d'une main humaine? Emblématique du pouvoir de prononcer, l'objet-livre doit se dérober à la connaissance, parfois pour un très long temps, puis être redécouvert pour nourrir la parole nouvelle. Somme toute, ce trajet qui va de l'écriture à l'oralité, laquelle ramène à l'écriture, apparaît comme une boucle mythique. Pour renvoyer à la réflexion de Roger Chartier dans sa leçon inaugurale au Collège de France, la peur de la perte et de l'oubli restera toujours liée à la prolifération incontrôlée de l'écrit, au désordre du discours, mais en ces temps-là du Moyen Âge, la lettre rare pouvait signifier et endiguer la peur de l'éphémère. Grimoire, armoire et mémoire : le geste de l'ouverture symbolique du désir, où la voix de l'origine s'enclôt sous les signes tracés, susceptibles de la ressusciter.

\section{BIBLIOGRAPHIE}

\section{Textes}

Coudrette, 1982, Le Roman de Mélusine ou Histoire de Lusignan. Texte édité par Eleonor Roach, Paris, Klincksieck.

Coudrette, 1993, Le Roman de Mélusine. Texte présenté, traduit et commenté par Laurence HarfLancner, Paris, GF-Flammarion.

Pierre Durand, Guillaume de Palerne. Texte édité par Annie-France Garrus (Thèse soutenue à l'Université de Bordeaux 3 Michel de Montaigne, non publiée).

Gérard de Nevers, Prose version of the Roman de la Violette, 1928, Texte édité par Lawrence F. H. Lowe, Princeton University Press ; Paris, PUF.

Histoire de Giglan filz de messire Gauvain qui fut roy de Galles, 1530, Imprimé par Claude Nourry, Lyon. Histoire de Gilion de Trasignyes et de dame Marie, sa femme, 1839, Texte édité par Oskar Ludwig B. Wolff, Paris, Brockhaus, Leipzig, Weber.

Jehan Wauquelin, 2002, La Belle Hélène de Constantinople. Texte édité par Marie-Claude de Crécy, Genève, Droz, p. 14.

Lais de Marie de France, 1990, Texte traduit, présenté et annoté par Laurence Harf-Lancner, édité par Karl Warnke, Paris, LGE, coll. Livre de poche « Lettres gothiques ». 
Lais féeriques des XII et XIII siècles, 1992, Présentation, traduction et notes par Alexandre Micha, Paris, Flammarion.

Lancelot, 1979, Édition critique par Alexandre Micha, t. IV, Paris, H. Champion, Genève, Droz. Lancelot en prose, 1488, Édité par Le Bourgeois, Rouen.

Le Conte de Floire et Blancheflor, 1980, Texte édité par Jean-Luc Leclanche, Paris, H. Champion.

Le Conte de Floire et Blancheflor, 1986, Traduit en français moderne par Jean-Luc Leclanche, Paris, H. Champion, coll. « Traductions des classiques français du Moyen Âge ».

Raoul Lefèvre, 1971, L'Histoire de Jason. Texte édité par Gert Pinkernell, Francfort-sur-le-Main, Athenäum Verlag.

Le Haut Livre du Graal, 2007, Texte établi, présenté et traduit par Armand Strubel, Paris, LGE, coll. Livre de Poche.

L'Istoire de tres vaillans princez monseigneur Jehan d'Avennes, 1997, Texte édité par Danielle Quéruel, Villeneuve-d'Ascq, Presses Universitaires du Septentrion, coll. « Bibliothèque des Seigneurs du Nord».

Le Roman de Floriant et Florete ou Le Chevalier qui la nef maine, 1983, Édition critique et annotée de la version en prose par Claude M. L. Levy, Ottawa, Éditions de l'Université d'Ottawa.

Le Rommant de Guy de Warewik et de Herolt d'Ardenne, 1971, Texte édité par Denis Joseph Conlon, Chapell Hill, University of North Carolina Press.

Les Prophesies de Merlin, roman en prose du XIII siècle, 2009, Édition critique par Nathalie Koble, Paris, Classiques français du Moyen Âge.

Robert de Blois, 1889-95, Roman de Beaudous, Robert de Blois. Sämmtliche Werke, Ed. Jacob Ulrich, Bd. L, Berlin, Mayer et Müller. [Rééd. Slatkine, 1978].

Le Romant de Richart sans paour, 1977, Texte édité par Denis Joseph Conlon, Chapell Hill, University of North Carolina Press.

Christine de Pizan, 2000, Chemin de longue étude. Traduction, présentation et notes par Andrea Tarnowski, Paris, Hachette, Livre de Poche, coll. « Lettres gothiques ».

Pierre Sala, 1953, Tristan : roman d'aventures du XvI ${ }^{e}$ siècle. Texte édité par Lynette Muir, Genève, Droz, Paris, Minard.

Roman du comte d'Artois, 1966, Texte édité par Jean-Charles Seigneuret, Genève, Droz.

Ysaÿe le Triste, 1522, Imprimé par Pierre Vidoue, Paris, pour Galliot du Pré (éd.).

Roman du comte d'Artois, ca 1550, Imprimé Jehan Bonfons, Paris.

\section{Ouvrages}

BAUMGARTNER, Emmanuèle, 1999, Du manuscrit trouvé au corps retrouvé, Le Topos du manuscrit trouvé. Études réunies et présentées par J. Herman et F. Hallyn, Louvain-Paris, Peeters, pp. 1-14.

BAUMGARTNER, Emmanuèle, HARF-LANCNER, Laurence (éds), 2002, Seuils de l'œuvre dans le texte médiéval, Paris, Presses de la Sorbonne nouvelle, 2 vol.

BOHLER, Danielle (dir.), 2005, Le Goût du lecteur à la fin du Moyen Âge, Paris, Éditions du Léopard d'Or.

BOUCHET, Florence, 2008, Le discours sur la lecture en France aux XIV et $\mathrm{XV}^{e}$ siècles : pratiques, poétique, imaginaire, Paris, Champion, «Bibliothèque du XVe siècle, 74 ». 
CERQUiGLINI-TOULET, Jacqueline, 2006, La scène de lecture dans l'œuvre littéraire au Moyen Âge, in Danielle Bohler (dir.), Le Goût du lecteur à la fin du Moyen Âge, Paris, Éditions du Léopard d'or, p. 13-26.

CHARTIER, Roger, 2008, Écouter les morts avec les yeux. Leçon inaugurale prononcée le 11 octobre 2007 au collège de France par le professeur Roger Chartier, Paris, Collège de France/Fayard.

CHARTIER, Roger, 2005, Inscrire et effacer : culture écrite et littérature XIe-XVIIIe siècles, Paris, Gallimard, Seuil, coll. « Hautes Études ».

CHINCA, Mark, Young, Christopher, 2005, Orality and literacy in the Middle Ages: essays on a conjunction and its consequences in honour of D. H. Green, Turhout, Brepols.

Coleman, Joyce, 1996, Public reading and the reading public in late medieval England and France, Cambridge, Cambridge University Press, coll. "Cambridge studies in medieval literature 26". GENETTE, Gérard, 1982, Palimpsestes, Paris, Seuil.

Goody, Jack, 1994, Entre l'oralité et l'écriture, Paris, PUF [The interface between the written and the oral, 1993].

Herman, Jan et Pelckmans, Paul (éds), 1995, L'Épreuve du lecteur : livres et lectures dans le roman d'Ancien Régime. Actes du VIII colloque de la Société d'Analyse de la Topique romanesque, Louvain ; Paris, Peeters, coll. «Bibliothèque de l'information grammaticale ».

KoBLE, Nathalie, ss presse, Les Prophéties de Merlin en prose : le roman arthurien en éclats, Paris, Champion.

KoBLE, Nathalie, 2006, Les enfances du livre : continuations à rebours d'une genèse romanesque, in Denis Hüe et Christine Ferlampin-Acher (dir.), Enfances arthuriennes. Actes du 2 colloque arthurien de Rennes, 6-7 mars 2003, Orléans, Paradigme, p. 127-142.

MANGUEL, Alberto, 1998, Une histoire de la lecture, Arles, Actes Sud [A history of reading, 1996].

RABEL, Claudia, 2006, «L'estude d'un tres noble seigneur garny a planté de pluiseurs beaulx livres ». L'iconographie des bibliothèques médiévales dans les manuscrits enluminés ", in Danielle Bohler (dir.), Le Goût du lecteur à la fin du Moyen Âge, Paris, Éditions du Léopard d'Or, pp. 245-289.

RÉGNIER-BOHLER, Danielle, 1989, « Pour ce que la memoire est labille » : le cas exemplaire d'un imprimeur de Genève, Louis Garbin, Le Moyen Français, Montréal, CERES, p. 187-213.

\section{NOTES}

1. Dans le Lai de Tydorel, la reine, pressée par la violence de son fils qui réclame le savoir de son origine, révèle le nom du père, le chevalier ondin, et elle ajoute que l'enfant à venir était contenu dans la parole même de son père, au moment où il adressait sa première requête amoureuse à la femme stérile (Lais féériques des XII et XIII ${ }^{e}$ siècles, 1992).

2. Voir aussi p. 139 : «Dans l'atelier d'écriture, le roman s'invente de nouveaux lendemains, puisque le clerc copie le texte inédit, raconte une partie de ce qu'il copie, ainsi que les séances de lecture qui en sont faites devant une petite assemblée de 'lecteurs errants', personnages nouveaux venus dans la fiction arthurienne ».

3. «[...] l'image du livre trouvé en l'isle d'Avalon en une sainte meson de religion qui siet au chief des Mares Aventurex résume la dualité irréductible de son inspiration. Mais dans le monde du lecteur les seuls vestiges tangibles, et pacifiés, de ce passé fascinant et inquiétant, sont l'abbaye et le 
codex : une 'maison de religion' dans les marais - une nature sauvage et inhumaine, mais destinée à être canalisée - qui ne sont plus qu'un toponyme gardant dans ses lettres toute la richesse d'un monde disparu, celui des vestiges et des muances d'une aventure sans fin, entraînée dans l'imbrication des vendettas et des crimes de sang; le livre, pieusement conservé dans un lieu sacré » (Strubel, 2007, 101-2).

4. L'ouvrage rassemble des contributions qui éclairent les mouvements de l'oralité et du scripturaire, le statut du texte écrit et la nature de la lecture «in an aural culture». Voir également « aural » et « aurality » dans Coleman (1996).

5. Prologue de Giglan : «Ung jour en une petite librairie [ou] j'estoiye trouvay ung gros livre de parchemin bien vieil escript en rime espaignolle assez difficile a entendre auquel livre je trouvay une petite hystoire laquelle me semble bien plaisante, qui parloit de deux nobles chevaliers qui furent du temps du noble roy Artus et des nobles chevaliers de la table ronde. »

6. Cette activité au sein d'un espace empli de volumes sera mise en parallèle avec l'abondance des représentations des bibliothèques dans l'iconographie de l'époque, voir l'article de $\mathrm{C}$. Rabel (2006, 245-291).

\section{RÉSUMÉS}

$\mathrm{Au}$ Moyen Âge, le livre manuscrit parle implicitement de la main qui lui a donné vie : le motif du livre, fréquent dans les narrations, confirme son statut de précieux témoin. Souvent caché dans une «armoire ", dans le secret du temps, le livre apparaît comme le fétiche énigmatique d'une culture éphémère. Lecture d'un livre entendu, ou livre issu d'une dictée ou encore livre trouvé en un lieu oublié, le manuscrit nourrit l'imaginaire de la transmission. De la voix de Merlin au livre dicté dans la forêt, du livre trouvé à la voix nouvelle du conteur, l'écrit est l'objet d'amour de celui que favorise le hasard, le «trouveur de livre ». Armoire rime avec mémoire : la découverte du livre est un événement symbolique d'un grand prix, et sa genèse est l'objet d'un rituel narratif très soigné. Les grains de poussière doivent être chassés pour faire place à un savoir dont s'enchante l'imaginaire. L'énigme de la parole enclose dans un espace oublié articule le seuil des récits : elle marque, comme une délivrance, le lancement d'une œuvre. Accéder au livre perdu signifie décrypter un secret. Y aurait-il un mythe de l'origine du livre, liée à la conjuration de l'oubli?

In the Middle Ages, the manuscript book implicitly speaks about the hand that gave it life: the motif of the book recurs frequently in narrations and confirms that it is a precious witness. Often hidden in a wardrobe, in the secret of time, the book appears like the enigmatic fetish of an ephemeral culture. A reading of a book that was heard, or a book that was dictated, or even a book found in some forgotten place, the manuscript nourishes the imaginary of transmission. From the voice of Merlin to the book dictated in the forest, from the book discovered to the new voice of a taleteller, writing is an object of love for the one who humors serendipity, the "book finder". Armoire rhymes with mémoire: The discovery of the book is a symbolic event of great worth, and its genesis is the object of a carefully produced narrative ritual. The grains of dust must be brushed off to give room to a form of knowledge with which the imaginary enchants itself. The enigma of words shut off in a forgotten place articulates the threshold of narratives: it marks, like a deliverance, the launching of a work. Reaching a forgotten book means unravelling a secret. Could there be a myth of the origin of the book, linked to the conjuration of forgetting? 
INDEX

Index géographique : Europe

Keywords : Europe, Manuscript, Reading, Medieval Literature

Thèmes : littérature

Mots-clés : livre médiéval, manuscrit, lecture, littérature médiévale 